Communication

\title{
Neuronal Coherence Agent for Shared Intentionality: A Hypothesis of Neurobiological Processes Occurring during Social Interaction
}

\author{
Igor Val Danilov ${ }^{1,}{ }^{*}$, Sandra Mihailova ${ }^{2}$
}

1. Academic Center for Coherent Intelligence, Riga, Latvia; E-Mail: igor val.danilov@acci.center

2. Riga Stradins University, Riga, Latvia; E-Mail: sandra.mihailova@rsu.Iv

* Correspondence: Igor Val Danilov; E-Mail: igor val.danilov@acci.center

Academic Editor: Bart Ellenbroek

OBM Neurobiology

2021, volume 5 , issue 4

doi:10.21926/obm.neurobiol.2104113
Received: October 02, 2021

Accepted: December 13, 2021

Published: December 29, 2021

\begin{abstract}
The present interdisciplinary study discusses the physical foundations of the neurobiological processes occurring during social interaction. The review of the literature establishes the difference between Intentionality and Intention, thereby proposing the theoretical basis of Shared Intentionality in humans. According to the present study, Shared Intentionality in humans (Goal-directed coherence of biological systems), which is the ability among social organisms to instantly select just one stimulus for the entire group, is the outcome of evolutionary development. Therefore, this interaction modality should be the preferred, archetypal, and most propagated modality in organisms, attributed to the Model of Hierarchical Complexity Stage 3. This characteristic of biological systems facilitates the training of the new members of the group and also ensures efficient cooperation among the members of the group without requiring communication. In humans, Shared Intentionality contributes to the learning of newborns. The neurons of a mature organism may teach the neonate neurons regarding the fitting reactions to the excitatory inputs of the specific structural organization. This enables the neonate neurons to develop a Long-Term Potentiation that links particular stimuli with specific embodied sensorimotor neural networks. The present report discusses three possible neuronal coherence agents that could involve quantum mechanisms in cells, thereby enabling the distribution of the quality of goaldirected coherence in biological systems (Shared Intentionality in humans). Recently reported
\end{abstract}

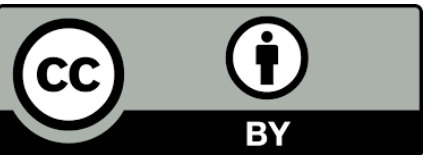

(C) 2021 by the author. This is an open access article distributed under the conditions of the Creative Commons by Attribution License, which permits unrestricted use, distribution, and reproduction in any medium or format, provided the original work is correctly cited. 
case studies conducted online with the task of conveying the meaning of numerosity to the children of age 18-33 months revealed the occurrence of Shared Intentionality in motherchild dyads in the absence of sensory cues between the two, which promoted cognitive development in the children. The findings of these case studies support the concept of physical foundations and the hypothesis of the neurophysiological process of social interaction proposed in the present study.

\section{Keywords}

Embodied cognition; goal-directed coherence; primary data entry; quantum brain; shared intentionality; social cognition; social interaction

\section{Introduction}

Piaget categorized the sensorimotor behavior development stage into four substages, each characterized by the development of new skills. According to Piaget, during the first substage named 'reflexes', an infant is just capable of manifesting pure reflexes [1]. In this stage, the goaldirected behavior is pure reflexes [2]. Many parents would confirm these considerations based on their experience with their children - intentional actions do not manifest in infants prior to the age of 2 months. However, this postulate of Piaget has been challenged by the social achievements of newborns under experimental conditions, where young infants have demonstrated varying social behavior, including imitation, facial recognition, recognizing the crying of other babies, etc. One could wonder how the neonates categorize a monolithic and uncategorized infinity to recognize a goal. For instance, in the case of imitation, how do neonates become sufficiently aware of their selfmovements, and how then, do they map these onto the movements of others $[3,4]$. According to Piaget, it is only in Stage III of secondary circular reactions that the cause and effect refer to the infant's self-action and its consequences, i.e., intentions.

These arguments appear to create a dichotomy. It could be that neonates utilize intentionality for the cognition of reality. However, intention is a conscious decision and is, therefore, not possible at the neonatal stage; i.e., the internal objectives of the infants could only be activated by the immediate environmental stimuli. In contrast, environmental stimuli, excluding those that correspond to primitive reflexes, are unintelligible and, therefore, unacceptable to infants. This dichotomy reflects the Primary Data Entry (PDE) problem. "Everything is known in comparison", as argued by René Descartes. Wittgenstein also claimed that the occurrence of communication requires a shared understanding of the meaning of the conveyed signal within a particular context among the community of users. That is to say, organisms require communication to begin communication.

\subsection{Primary Data Entry Problem}

According to Danilov and Mihailova [5], the existing understanding of cognition has established three main concepts or approaches within cognitive science - cognitivism, connectionism, and embodied dynamicism. Several theories within this framework study the onset of cognition. The interesting ones among these theories include the cohort of Embodied dynamic system theories [6], 
the theory of innate intersubjectivity and innate foundations of neonatal imitation (the theory of the development of human communication) [7], the theory of natural pedagogy [8], and the theory of sensitivities and expectations [9]. However, despite these various theories, there remains a gap in the knowledge regarding cognition, in the form of the Primary Data Entry (PDE) problem.

According to the embodied dynamic system approach (which is relatively closer to solving the problem), intelligence emerges when an organism interacts with an environment due to sensorymotor activity. This approach tends to solve the above-stated gap by introducing the concept of dynamically embodied information [6]. The descriptions of embodied cognition might be organized around a larger number of narrower themes [10], while the effort to broaden the themes could reduce their number, risk of generalizing the description of embodied cognition to the extent that its purported novelty is jeopardized [10]. Context dependency is one of the principles of this approach - the embodied dynamic system theories follow Vygotsky's concepts of arising cognition through social contexts [11]. Therefore, prior to introducing the embodied dynamic system concept, it is necessary to explain the categorization of reality through intentionality. According to the embodied cognition approach, symbols encode the local topological properties of neuronal maps [6], which reflect a dynamic action pattern. The sensorimotor motor network enables the pairing of the binary cue stimulus with a particular symbol saved in the structures and processes that embody meanings. 'Representational "vehicles" are temporally extended patterns of activity that may crisscross the brain-body-world boundaries, and the meanings or contents these vehicles embody are brought forth or enacted in the context of the system's structural coupling with its environment [6] (p. 36)'. Therefore, the embodied cognition approach requires introducing the mechanism of intentionality prior to cognition. In a multi-stimuli environment, the stimulus-consequence pair is unpredictable due to the several irrelevant stimuli that claim to be associated with the embodied dynamic information randomly. The bond of the stimulus-consequence pair related to a social phenomenon in the sensorimotor network requires the categorization of the reality by the nervous system prior to applying the innate reflex regarding this social phenomenon to a specific case. Therefore, dynamically embodied information is applicable only if intentionality is already in place. This would imply that cognition requires intention, which is absent at the beginning of cognition. Therefore, a vicious circle is created - the pure mind already requires meanings to acquire the first meaning. However, at this stage of development, organisms are unable to exhibit cooperative actions with their co-mates through sensory cues as these cues are unintelligible.

Interestingly, recent hyper-scanning research has revealed coordinated neuronal activities in individuals during cooperative actions in the absence of communication via sensory cues [12] and a greater interpersonal neuronal coordination in subjects involved in solving a problem together compared to the individuals working separately on identical tasks [13]. Another recent study conducted with adults demonstrated that coordinated actions between unprimed individuals and primed confederates could facilitate resolving unintelligible problems without requiring communication on the basis of sensory cues provided by those confederates who knew the correct answer [14-16]. Case studies that involved assigning educational tasks to children aged between 12 months and 33 months revealed the occurrence of Shared Intentionality in the absence of communication via sensory cues in mother-child dyads, which then promoted numerosity in the infants and toddlers within a short duration and at an age younger than others (earlier compared to the peers) $[17,18]$. 
The present report discusses the physical foundations of the neurobiological process occurring during Shared Intentionality in humans (goal-directed coherence of biological systems), which includes what agent (element or compound) inside the cells is involved in the cooperation and the underlying mechanism. In addition, the role of social interaction without sensory cues in resolving unintelligible problems is analyzed by integrating the findings of neuroscience and physics. The remaining portion of the present report is organized as follows. Section 3 provides a review of the coherence in different biological systems. Section 4 discusses the property of Shared Intentionality by opposing intentionality and intention from both theoretical and empirical perspectives. The question of how the brain learns is based on understanding the physical laws that the brain obeys. In this context, Section 5 discusses the physical foundations of Goal-directed coherence in biological systems, comparing three potential candidates for the agent of quantum mechanisms occurring within the cells. In Section 6, the hypothesis of neurophysiological and physical foundations of Shared Intentionality is detailed. In the last section, a summary of the complete report is provided.

\section{Methods}

Understanding the above-stated problem requires analyzing social interaction in different species. In particular, the knowledge of how simple organisms interact is required for understanding the possible social interactions among humans. In this context, the present study analyzed the interactions in different biological systems at the developmental stages corresponding to those of human fetuses and infants. The Model of Hierarchical Complexity (MHC) [19] effectively compares the various biological systems [that appeared at different time points in evolutionary history] in terms of their information processing capabilities. In the MHC, the order of hierarchical complexity of a task is quantified based on how the information is organized in organisms [19]. In this manner, this mathematical model allows comparing the cognitive development of different species that are at different stages of their development. The determination of the relationship between development and evolution and the comparison of interactions among different species based on the MHC is facilitated by three arguments. The first argument is based on the fact widely accepted across various theoretical approaches that there are common mechanisms of molecular genetics underlying the development and evolution of all morphological forms in multicellular organisms. The second argument is that a genetic comparison between species has become possible recently, which has demonstrated their relationship. The third argument is that such a comparison is possible as information processing could be an indicator of cognitive development, and the $\mathrm{MHC}$ is based on principles of information processing that rely only on the modalities of interactions and not on the biological characteristics of the subjects. For instance, from this perspective, information processing in insects is associated with Stage 3 of the MHC, which is comparable to the fetuses at the sensorimotor stage of development described by Piaget. According to Danilov and Mihailova [20], the comparative analysis of social interaction in newborns of different species, whose behavioral development corresponds to the circular sensorimotor Stage 3 of behavior development, demonstrates that organisms are capable of distinguishing identical stimuli through their significance without requiring perceptual driver stimuli.

The development of advanced digital modes of learning requires an understanding of the brain function at the cellular level during social interaction. The present study, therefore, attempted to address this concern by investigating a two-fold objective: (1) Understanding the physical 
foundations of interaction within groups, comparing different species that are at the same stage of development (at the MHC Stage 3 in animals and humans, which corresponds to the development of the organisms up to and including Piaget's sensorimotor stage fetuses and infants), and solving the PDE problem in the absence of sensory cues. (2) Describing the ability of an organism in a group to act intentionally without Intention-Shared intentionality; i.e., to define the agent and the neurophysiological process that could enable such interaction in the absence of sensory cues.

This approach might be able to define the modality of interaction in biological systems in the absence of communication via sensory cues capable of facilitating communication and knowledge acquisition and providing a human-computer interface design for advanced Artificial Intelligence Systems.

\section{Coordinated Activity in Different Biological Systems}

Bacteria are known to exhibit a unique characteristic of coordinated motility in colonies. These smallest and the most ancient relatives of humans exhibit goal-directed coherence within a particular electromagnetic field, which is the ability of social organisms to select just one stimulus for the entire group instantly. Under specific conditions, different biological systems manifest goaldirected coherence (in the absence of sensory cues) in one form or another. The present review briefly highlights the common characteristics of this quality in different species.

\subsection{Bacteria}

These small free-living (self-replicating) organisms were among the first life forms to appear on Earth. Under the influence of an electromagnetic field, free-swimming bacteria change direction and move together in a favorable direction. The phototaxis mechanism in bacterial populations challenges the existing knowledge regarding the interactions demonstrated within a bacterial colony as no evidence supports that individual bacterial cells are capable of solving this problem using only receptors [21]. The mechanism underlying the directional light perception in bacteria remains to be deciphered so far [21]. It is hypothesized that certain bacteria might rely on detecting temporal changes in the light environment [21]. However, this hypothesis does not appear to be universal as different bacteria recognize different electromagnetic field gradients differently by measuring the intensity and the spectral quality of the light sources [21]. Another hypothesis is that individual bacterium acts as a highly efficient lens that focuses light at the edge of the cell away from the light source [21]. However, these explanations of the demonstrated community phototaxis in bacteria are debated in the biology and physics literature [21]. Moreover, the ability of individual organisms to independently determine the direction of movement also contradicts the simplicity of their internal structural organization [21].

\subsection{Earthworms}

According to Zirbes et al. [22], earthworms demonstrate the cooperative ability to select the same direction of movement as their conspecifics. Experiments in a binary choice test revealed that contacts between individuals are responsible for the demonstrated collective movement [22]. The ability of individual organisms to move in the same direction demonstrates the incongruence of the complexity-requiring communication and a set of sensory receptors (or sensory modalities) as the 
simple nervous system and reduced sensory modalities in earthworms render any communication impossible [21]. So far, no hypothesis has been proposed for how these organisms solve the problem using just receptors.

\subsection{Ants}

Darwin noted a navigation system in animals - the Path Integration (PI). Individual ants perform large distance foraging excursions of up to $1200 \mathrm{~m}$, and while returning, the ants select a direct shorter path back to their nests and are capable of inferring this ground distance when walking over hills [23]. These organisms determine the actual navigation strategy from complemented and contrasted navigation mechanisms with different weights that are based on their reliability. The ants appear to select these mechanisms through interaction with their nest-mates on a case-bycase basis [21]. However, their perceptual capacity and other characteristics required for successful interaction-reasonable distance and appropriate environmental conditions should not allow the ants to complete this operation [21]. These organisms solve the problem in cooperation with their co-mates regardless of the capabilities of their receptors and the distance from them.

\subsection{Bees}

Bees are capable of transmitting route information to multiple destinations. Buatois and Lihoreau [24] worked with arrays of feeders and demonstrated that honeybees could learn complex foraging circuits integrating a minimum of five different locations. Quorum decision-making is another characteristic of bees, which is beyond their interaction ability. Bees are not able to communicate via symbols at their development Stage 3 of MHC [21]. The only possibility for the bees is the interaction through binary cues. The main disadvantage of such interaction is the large number of binary signals required for encoding information and the complexity involved with the decoding of these binary signals [21]. Therefore, this encryption system requires a huge memory capacity. Even spatial parameters contain several binary signals and, therefore, information regarding site significance also requires higher memory [21]. Therefore, interaction among bees requires a highly-developed processing mechanism with a large memory [21]. This circumstance does not correspond to the existing knowledge regarding the behavioral development in insects, which corresponds to Stage 3 of the MHC. This ability of organisms to undertake a decision in a quorum and their cooperation in complex foraging circuits proceed independently from their receptor ability and the distance from their co-mates [21].

\subsection{Human Fetuses}

Fetuses are capable of demonstrating social behavior without any sensory link with social reality. Fetuses do not exhibit social behavior independently due to a lack of understanding of the social reality [25]. The link between a particular social situation and the corresponding social expression, therefore, is a problem for human fetuses. The connection between the fetal nervous system and the social reality, which allows the embodied sensorimotor networks to be activated, is undeveloped. Moreover, the disadvantage of their physiological development during pregnancy also hinders their ability to behave socially. If fetuses could behave socially, their social skills would not appear from self-learning, because if they could exhibit a range of behaviors, the innate 
endowment would have to be more complex than a couple of reflexes [25]. Finally, according to Danilov [25], the social behavior of fetuses emerges from and is guided by mental collaboration with the mother. In the mother-fetus dyad, this cooperative performance of organisms succeeds in the absence of communication through sensory cues; it must succeed as no advantage of stimuli association is possible without a preference of inputs in a noise environment [25].

\subsection{Human Infants}

The growing evidence in favor of facial recognition, other-race effect, and imitation in newborns, and that for word categorization in infants, confirms their social behavior [20,26]. Infants, and even newborns, successfully classify social phenomena that are abstract or absent from their reality [26]. Their ability could emerge only from the mother-child connection, which succeeds in the absence of communication based on sensory cues. Recent experiments have demonstrated that the emotional contagion which assists the infants in acquiring their initial social phenomena could appear through body language cues that are not perceived consciously by the subjects [20].

\subsection{Discussion}

The analysis of social interaction in various species revealed the common characteristic of goaldirected coherence, which is the ability of the organisms within a group to be able to distinguish identical stimuli according to their significance, enabling them to select just one stimulus for the entire group. Accordingly, the main characteristics of goal-directed coherence could be as follows: bypassing sensing (insensitivity to sensory perception), independence from a distance, and instantaneousness in time [20]. These features of goal-directed coherence are consistent with only the quantum mechanical approach. Common sense indicates that the organisms in developmental stages prior to Stage 3 of the MHC would prefer interaction without sensory cues, suggesting this kind of interaction to be the oldest and the most propagated one. This quality of biological systems ensures the training of the new members within the group and also higher cooperation efficiency in colonies without requiring communication. The bonds within a colony offer the advantage of sustainable development of the organisms in contrast to the survival of single individuals. The former contributes to preserving and propagating the corresponding phenotypic qualities in organisms. In higher complex organisms, goal-directed coherence should manifest itself in one form or another as it has been preserved in simple organisms. Therefore, it could be assumed that the behavior of neurons in a mature organism governs (or trains) the neurons of a newborn through a certain mechanism. According to the above arguments regarding the relationship between development and evolution when comparing the interactions in different species (refer to the Introduction section for details), since biological systems tend to exhibit target-directed coherence, the same quality could appear in humans as well.

\section{Intention vs. Intentionality}

In humans, learning in newborns (in the absence of communication that is based on sensory cues) begins with Shared Intentionality [27]. The present study investigated Shared Intentionality by opposing intentionality and intention from both theoretical and empirical perspectives. Intentionality and intention, the two agitators of learning, emerge sequentially, i.e., learning 
emerges in humans from the unaware cognition in Shared Intentionality, and later, learning becomes conscious (at least in part), following (and due to) the gradual increase in awareness. This implies that at the onset of cognition, organisms learn due to Shared Intentionality, and then, at a further advanced stage of increasing awareness, learning is based on both intention and intentionality; i.e., in mature organisms, intention and intentionality go together.

\subsection{Theoretical Approach to Intentionality and Intention}

Since language continues to evolve, its plasticity and adaptability to increasing knowledge require the semantic competence of researchers to employ a word's meaning corresponding to its actual usage. The latter also implies an analysis of the corresponding empirical data in the literature to the modality of this phenomenon. Intentionality and intention are separate concepts as these bear different semantics and reflex different appearances. The fact that these two terms have the same etymological root is coincidental; Searle [28] (p. 3) referred to this as a "pun" [2]. The present study, therefore, investigated Shared Intentionality by studying and opposing intentionality and intention from both theoretical and empirical perspectives.

According to Searle [28], intentionality is the directed property of certain mental states, while intentions are the causal antecedents of action. According to the accepted view, the intention is a conscious manifestation, i.e., 'a prior conscious decision to conduct a behavior' [29]. Lewis [30] raised a question regarding intention development, asking how to go from the absence of the mental state, from intention to its presence; i.e., 'where does intention arise from?' [30] (p. 233).' However, this question was not accurately developed either in the work of this author or in the subsequent related studies conducted by other researchers. For instance, Lewis did not consider the difference in the meanings of these two terms (intentionality and intention) [2]. The subsequent arguments in the present study would be focused on discussing why this probably happened. Lewis [30] attempted to solve the problem of the appearance of intentional actions by proposing that all goal -directed systems are intentional from the beginning. Bargh [31] deduced the equation of dependency between intention and the desire for the target (what corresponds to the modern understanding of the term) by analyzing Lewis' thought; the equation is provided below:

$$
\text { Intention = Goal - directed behavior }+ \text { Desire for the goal }(I=G+D)
$$

This explanation is correct for the organisms at the developmental stage where children are already able to create targets and plans in the absence of external events directly relevant to these targets and plans. However, according to the literature and common sense, organisms in the reflex stage do not manifest conscious decisions. The desire for a goal warrants self-awareness as a minimum requirement and also the understanding of social reality. Fodor [28] highlighted the same issue by asking how could children learn a new concept unless they already had the ability to hypothesize the concept. According to Lewis [30], in the reflex period of the sensorimotor stage, 'the action, although intentional, is both predicated and prescribed by survival' (p. 241); here, the definition of intentional action within the sense of intentionality has been employed. The reflex actions of newborns do not reflect the desire for a target as they do not contain and bear the causal prerequisites for actions. Therefore, these actions cannot be attributed to intention, although these are intentional in the sense of intentionality. These actions must be intentional because of the evident success in the continuous development of goal -directed biological systems. 
Nowadays, intentionality is generally defined as a characteristic of an individual's acts that requires the individual (a) to have goals, desires, and standards, (b) to select behaviors that are in the service of attaining the goal (e.g., means to an end), and (c) to call into conscious awareness a desired future state [29]. Common sense dictates that, unlike intention, intentionality may manifest without the desire and awareness of the individual or unconsciously as well. For instance, people could be unable to stop thinking regarding a traumatic life event and would think obsessively regarding the event to the point of distraction and an inability to function in their daily life [31]. This implies that, while intentionality manifests consciously, a part of this process is not occurring consciously.

These perspectives indicate that there is no contradiction as to whether or not the actions of neonates are intentional. Although the young infants employ intentionality for the cognition of reality (and they must do this for continuous development) from the beginning of life, they cannot independently desire a goal by performing an intentional behavior on their own, i.e., newborns do not demonstrate intention. Therefore, it could be assumed that the ability of intentionality is possible because neonates, being with their caregivers, may also manifest intentionality unconsciously, without their desire and awareness.

This reflection highlights the unconscious part of intentionality, which is the basis of it being distinguishable from intention. Searle [32] described a unique part of intentionality, referred to as Shared Intentionality, which is a social bond appearing during social encounters. Later, Tomasello $[27,33]$ applied the concept of Shared Intentionality in solving the problem of the beginning of cognition based on the embodied dynamic model. Tomasello [33] argued that the gradually increasing social bond development in children referred to time slices: (1) sharing of emotions since birth, (2) joint intentionality from the nine-month revolution, (3) collective intentionality at approximately three years of age, and finally, (4) reason and responsibility. According to Tomasello [33], the beginning of cognition appears through the newborns' basic motive force of Shared Intentionality. Notably, this protoconversation based on reading (interpreting) the emotional expressions of others is impossible for infants if it is only in terms of sensory interaction. According to Danilov and Mihailova [15], the mechanism underlying such coordination of emotions remains unclear as it is based on the sharing of emotional expressions. So far, there is no evidence of a genetic mechanism linking the meaning in the mind with a certain social reality to apply an appropriate emotional neural pattern to a specific situation [24]. That is, innate neural patterns of primitive emotions cannot be associated with explicit bodily expressions that bear a correspondence between a specific psychophysiological state of the individual and particular social reality. The central point here is the link between the specific psychophysiological state of the individual and a particular social reality that is inaccessible to the newborns. Neonates are unable to understand the meanings of the emotional expressions of others. Therefore, the protoconversation cannot proceed through the interpretation of emotional expressions. Therefore, this interaction is neither conscious nor perceptual. The deductive reasoning concludes with the postulate of the foundations of intentionality - the non-perceptual interaction. The authors attribute this ability to the property of shared intentionality.

In consideration of the above reflection on shared intentionality, it is possible to suppose that the unconscious part of intentionality participates in the formation of intentionality:

Intentionality = Goal-directed behavior + Desire for the goal + Shared-Intentionality $(I=G+D+$ Si $)$. 
The desire for a goal is commonly referred to as motivation. Therefore, this dependence could imply that in newborns, Shared Intentionality could result in motivation.

Danilov [34] argued that Shared Intentionality is the essential quality of organisms contributing to the survival advantages of humans. The paradigm that humans only have a sensory perception of the intentionality of others is challenged by increasing evidence from research. According to Danilov [34], the analysis of biological systems highlights the qualities of goal-directed coherence, which should be the same for Shared Intentionality in humans -immediacy in time, independence from a distance, and insensitivity to sensory perception.

According to the literature, which grants a theoretical perspective, the difference between intentionality and intention manifests as Shared Intentionality. The following sections of the report discuss the investigation on Shared Intentionality from these perspectives by exploring the research works concerning brain-to-brain neuronal synchronization based on intentionality, intention, and motivation as the research queries. The model of Shared Intentionality is also observed in the section - the neurobiological foundation of Shared Intentionality.

\subsection{Empirical Approach to Shared Intentionality}

According to Schirmer et al. [35], an increasing number of studies are being conducted on psychophysiological and brain activity coordination to measure interactional synchrony. These hyper-scanning research paradigms differ in whether they elicit intentional or unintentional synchronization. A few examples of the former are music composing or tapping, while passively observing others or engaging in conversation are examples of the latter [35]. These two paradigms demonstrate the integrated outcome of social interaction, including a spectrum of stimuli ranging from symbolic to physical interactions. Even the awareness of the presence of others could alter the mental state of the subjects, affecting their neural activity. This implies that hyper-scanning could enable observing Shared Intentionality (the difference between intentionality and intention) if other interactions could be reduced during experiments. Therefore, the present study reviewed hyper-scanning studies conducted on cooperative mental activity without sensory cues-based communication between the subjects.

Two meta-analyses of hyper-scanning studies, the review on 24 studies involving EEG-and fNIRS hyper-scanning methodologies [36] and the neuroimaging meta-analysis of 50 monetary incentive delay task based fMRI studies [37], included reports on coordinated neuronal activity during cooperative mental actions. According to Danilov and Mihailova [5], only 4 studies among all investigated excluded sensory interaction between subjects. These studies demonstrated that phase synchronization appears in a similar manner across different brains during meaningful social interaction. According to Valencia and Froese [36], evidence of inter-brain synchronization in the fastest frequency bands overcomes the most convincing skeptical position to date.

However, even though these 4 studies involved the subjects performing identical tasks without communication, their outcome could not be considered pure Shared Intentionality as the subjects had knowledge of social encounters during the experiments. Therefore, rather than mental collaboration, their results could simply reflect increased brain activity due to similar emotional arousal in the participants stimulated by the social encounter. The review of the literature in the present study revealed several recent works that were not included in the above-noted reviews. One among these was a fascinating study on interaction in dyads conducted with 12-month-old 
infants. According to Wass et al. [38], while engaged in free-flowing naturalistic parent-child play, parents exhibited an oscillatory activity recorded over the frontal areas that varied with their respective infants' attention patterns, independent of the former's attention patterns. In addition, weaker evidence for the opposite relationship, i.e., the infants' brain activity being in sync with the adults' attention patterns, was observed [38].

Another recent study used electroencephalography (EEG) to record the human ability to coordinate actions in the absence of sensory cues [12]. The experiment demonstrated the interbrain synchronization among different individuals, which probably implied their engagement in Shared Intentionality. While this outcome supports the hypothesis of insensitivity of sensory perception to Shared Intentionality, additional experiments on hyper-scanning are nonetheless warranted, for example, with various mental tasks [14-17]. As noted above, the notion that humans are only capable of sensory perception of the intentionality of others is challenged by increasing evidence from the research conducted in disciplines other than neuroscience. Therefore, it would not be strange to evaluate this concept in a hyper-scanning study.

The design of the future hyper-scanning research should eliminate other interactions from the experiments conducted on Shared Intentionality. The authors propose two conditions for such a research design: intentional synchronization and unintentional synchronization, without communication between the subjects in both cases. It appears that the new paradigm could enrich the experimental data on interpersonal synchrony and thereby answer several questions investigating the beginning of cognition. In summary, further hyper-scanning research on interbrain synchronization is warranted to explore intentions in subjects under the above-noted integrated paradigm, without any social interaction between the participants.

\subsection{Discussion}

The observation of intentionality and intention reveals the basis of intentionality, i.e., the nonperceptual interaction, the Shared Intentionality. One could then ponder whether Shared Intentionality bears unconscious and even a non-perceptual impact. In recent research, progressive neuronal recruitment prior to the demonstration of intentional action by subjects [39, 40]. The mechanism underlying intentionality probably involves deep (and even unconscious) levels of thought processing. Then, one might question whether this process could bypass the receptors and sensorimotor networks via connecting the different nervous systems directly? The authors of the present study believe that the property of Shared Intentionality is the evolutionary outcome of the quality of goal-directed coherence demonstrated by simple organisms, which promotes the survival advantages of biological systems, and, of course, social interaction is subordinate to physics laws.

\section{Physical Foundations of the Goal-Directed Coherence}

The understanding of the physical foundations of Shared Intentionality (goal-directed coherence in other simpler biological systems) raises a question regarding which of the physics laws promote the direct connections between the neuronal circuits of different organisms. An essential argument of the present is a consensus on the terms and definitions used in neuroscience and physics. The literature includes several studies from the field of neuroscience which use the terms of resonance and coherence for the recorded activation of neurons in experiments conducted using different techniques, such as Nuclear Magnetic Resonance Imaging (NMRI), Functional magnetic resonance 
imaging (fMRI), Electroencephalography (EEG), Functional Near-Infrared Spectroscopy (fNIRS), and Magnetoencephalography (MEG). While all of these techniques are efficient in observing the activity of neurons, the detection in these techniques is just indirect evidence of resonance and coherence of neurons, which implies that these techniques assume neuronal stimulation that cannot prove the resonance and coherence of the neurons. For instance, in the MRI technique, a radio frequency signal of the resultant evolving spin polarization is detected by certain atomic nuclei that are capable of absorbing the radio frequency energy when placed in an external magnetic field. Hydrogen atoms are used the most frequently for generating a macroscopic polarization and, therefore, most MRI scans essentially map the location of water and fat in the body. Another technique is the fMRI, which measures brain activity by detecting changes associated with the blood flow. Cerebral blood flow is associated with neuronal activation. When a region in the brain is activated, the blood flow to that region increases. EEG is a method of electrophysiological monitoring to record the electrical activity occurring on the scalp, with the aim of detecting the macroscopic activity of the surface layer of the brain underneath. The fNIRS technique detects brain activity using the near-infrared light for assessing the cortical hemodynamic activity, which occurs in response to neural activity. The MEG technique records the magnetic fields produced by the electrical currents generated naturally in the brain.

While our knowledge regarding consciousness is improving with the study of interactions among the neurons, modern tools are unable to directly measure the dynamics of the electromagnetic activity of single neurons within the subjects involved in cooperative efforts with other individuals. Therefore, currently, the reasoning regarding neuronal resonance and coherence remains speculative when using the neuron activity data from the MRI, fMRI, EEG, fNIRS, and MEG techniques. It is difficult to estimate the resonance and coherence of neurons using the data from the above-stated techniques. However, due to the lack of other resources, these techniques appear to be the best ones for current use, while continuing the research for developing better techniques. The text in this paragraph is focused on the terms assigned to the phenomena. Since neuroscience applies physical tools to measure psychophysiological phenomena, the present report uses physical terms that describe the processes in the detection techniques applied in neuroscience. This is particularly because neuroscience studies neurons as physical objects even if the dimensions of these neurons are similar to the objects studied at the quantum mechanics scale [5]. In physics, the term resonance describes the increase in the system's amplitude when both frequency and phase of the applied oscillator are equal to the natural frequency and phase, respectively, of the system on which the former system acts. Coherence in physics implies an identical waveform.

In classical physics, all matter with a temperature greater than absolute zero emits thermal radiation which comprises electromagnetic fields propagating through space. Since coherence reflects a fixed relationship between the phase of the waves in a beam of radiation of a single frequency, two neurons are coherent in the case when their characteristics correspond to their thermal radiations. According to quantum mechanics, all particles have wave-like properties. The quantum mechanics theory argues that particles are conceived as having the property of waves when isolated from their environment, while their wave function is described as collapsing into a particle when brought into contact with the environment through a process of decoherence. Quantum coherence appears even upon the interference of two quantum waves of the same particle. Quantum coherence is a condition necessary for both entanglement and other types of quantum correlations. Therefore, the argument in the present study is a consensus regarding the 
terms and definitions in neuroscience and physics. At both macro and micro scales, neurons are a source of electromagnetic waves and a subject of the coherence of these waves. The present report, therefore, uses the term coherence only in the case of identical waveforms (wave function) of two or more neurons, while the synchrony of the registered activity of neurons is referred to as coordinated neuronal activity. Moreover, the term entanglement (or quantum entanglement) is applied only in terms of physics and is not used for referring to the simple synchrony of the registered activity of objects. The term quantum entanglement implies that the objects interact such that the quantum state of each cannot be described independently of the state of the others, including the case when a significant distance separates the particles. The present report adheres to such requirements even if the source of information states otherwise. The consensus on these terms is an essential part of the current analysis.

\subsection{Coherence Agent}

An increasing number of studies on quantum consciousness have presented numerous theories that deal with the question of quantum effects in the brain and the underlying mechanisms involved. Recent discussions and findings in the field of quantum mechanics have demonstrated that the quantum approach should be considered in understanding the functions of the brain. 'In sum, it is now well-established, despite the commonly held view, that quantum effects cannot be present only in mammalian brains and that quantum phenomena are indeed quite common in the biological systems [41]'. It is clear that the concept of physical foundations and the hypothesis of the neurophysiological process of social interaction, both require an agent with the size range of the quantum scale, which introduces quantum mechanics in the cells and propagates the quality of goal-directed coherence in different species. Without such an agent, further research on quantum consciousness and the physical foundations of social interaction remains limited. Therefore, the section ahead discusses three possible coherence agents that could involve quantum mechanisms in the cells, thereby propagating the quality of goal-directed coherence in biological systems and promoting Shared Intentionality in humans. These agents are as follows: the atom of hydrogen, the Posner molecule, and protein.

\subsection{Protein}

Proteins become biologically active only when these occur in a three-dimensional structure formed of amino acids folded into particular highly complex configurations [42]. Proteins fold into their functional configurations at a rapid rate, with a relatively small protein of only 100 amino acids completing this process within nanoseconds [43]. Only quantum mechanical mechanisms are capable of achieving such a high rate of selection from a huge number of options (10 to the power of 100 different possible amino acids configurations!) [43]. The high rate of the shaping of the protein molecules and the symmetric configuration of the protein structures support the hypothesis of amino acid relationships in quantum mechanisms [43-47]. The protein molecules are born, and their amino acids are connected within and between molecules under the laws of quantum mechanics. Evidently, quantum mechanisms are not limited to the structure of a single protein molecule. The protein molecules play essential roles in photoreceptors, which regulate motility in bacteria, thereby contributing to the community phototaxis in bacteria [48]. The review on plantassociated bacteria reported the observation of protein-protein interactions between the 
photoreceptors in bacteria during signal transduction which was identified as a common trait for all [49]. Photoreceptors interact with each other and mutually modulate their individual effects [49]. Moreover, the photoreceptors of plant-associated bacteria maintain their cooperation with their plant hosts under control, i.e., these light-sensitive proteins appear to control infectivity and virulence to the extent that does not generate much harm to their host plants [49]. This could lead to a speculative conclusion regarding the non-local relationships between amino acids of the bacterial proteins and those from the host plants, which could only be relevant in regard to quantum mechanisms.

Therefore, it appears that quantum mechanisms regulate the protein-protein interactions within a bacterial colony and those between bacteria and plants. In humans, the protein Reelin is essential for hippocampal integrity and synaptic plasticity. According to Faini et al. [50], this molecule contributes to the neural circuit assembly, refinement, and function. In addition, accumulating evidence indicates a significant role of Reelin in axonal guidance, synaptogenesis, and dendritic spine formation [50]. The entanglement between the protein molecules of neurons in different organisms could be the connection mechanism that leads neurons of an immature organism to respond appropriately to stimulations, similar to those demonstrated by the teacher receiving the same stimulations.

\subsection{Posner Molecules}

Fisher [51] introduced an elegant hypothesis regarding a molecular process that could promote quantum entanglement between neurons. Phosphorus is present in several biological substances. According to Fisher [51], quantum entangled Posner molecules appear due to the enzymatic hydrolysis of extracellular pyrophosphate, a process in which phosphorus atoms might be in a quantum entangled singlet state. The presence of entangled Posner's molecules in the cytoplasm of multiple presynaptic neurons could lead to post-synaptic firing that is quantum correlated across these neurons. When Posner molecules in different neurons are entangled, these incur binding reactions and hydrolysis, which may lead to the release of calcium-mediated glutamate from presynaptic neurons and subsequently non-local quantum correlations in post-synaptic firing [51].

\subsection{Hydrogen}

Hydrogen bonds are weak, generally intermolecular bonds, which hold most of the soft matter together and also the condensed phases of water and network liquids [52]. The small mass of hydrogen implies that hydrogen atoms are inherently quantum mechanical in nature [52]. According to the quantum electrodynamics field theory, water is quantum coherent under ordinary conditions [53, 54]. According to Dirk K. F. Meijer et al. [55], water functions as the primary antenna (mirror) for external electromagnetic field influences and is able to transmit these vibrations to other dissolved substances in a manner that, in unison, coherent vibration domains of cell compartments are formed. Since water is present everywhere, this concept proposes that thoughts and consciousness emerge from quantum superfluids, from where all information is poured in [56]. 


\subsection{A Few Considerations regarding the Agent}

A few comments would contribute to establishing a basic framework for conducting further research on understanding what shapes consciousness and fosters goal-directed coherence. The first comment is regarding the protein agent hypothesis, which appears to be a more plausible concept based on the above discussion. Protein molecules could serve as candidates for the role of an agent that engages neurons of different organisms in cooperative reactions to similar stimuli. Further investigation is, however, required to determine whether these protein molecules are involved in the mechanisms of coordinated action in all biological systems or only for certain species, specifically just as the cases discussed above.

The second comment regarding the elegant hypothesis of the Posner molecule, which provides a plausible explanation of the quantum entanglement mechanism of neurons. This concept is challenging to apply to goal-directed coherence in biological systems, for example, in bacteria. Let us assume that the organisms with a nervous system and those without one have different mechanisms contributing to goal-directed coherence. However, according to empirical data, the application of the Posner molecule properties to quantum mechanisms in organisms with a nervous system appears questionable. Recently conducted extensive analysis of the dynamical and structural properties of Posner molecule using over thousands of sampled configurations suggests that this molecule exists predominantly in low-symmetry molecular structures, such as $\mathrm{Cs}, \mathrm{Ci}$, and C1, at room temperature [57]. However, highly-symmetric Posner clusters are reported to be essential for supporting the quantum biological hypotheses [57].

The third comment is from the perspective of propagation in nature, according to which the hydrogen concept postulated by Dirk K. F. Meijer et al. [55] appears to become further intriguing relative to the other ones discussed in the present report. While this concept overcomes the traditional approach of complementing additional properties to a classical neural network property to proclaim quantum characteristics in the brain [56], it is too metaphysic to agree with the laws of physics. Investigation revealed that it is complicated to reduce the influence of other factors on the object to detect pure "quantum superfluids" (in the case these exist) in the object's environment, which entails gravity, electromagnetism, strong nuclear force, and weak nuclear force. Furthermore, even if the pure "quantum superfluids" exist, why has their presence not been perceived so far.

\subsection{Electromagnetic Field may Induce Quantum Entanglement in Different Atomic Systems}

At the cellular level, the brain function obeys physics laws. The brain function should also obey the laws of quantum mechanics as the dimensions of the essential components of neurons are similar to the objects studied at the quantum mechanics scale [5]. In physics, it is generally accepted that coherence could be transformed into entanglement; conversely, each entanglement measure corresponds to a coherence measure. Accordingly, any non-zero amount of coherence in a system could be converted into an equal amount of entanglement between that system and another initially-incoherent system [58]. Coherence appears even at the atomic level and occurs widely along with the electromagnetic field. Atomic coherence is the induced coherence between the different levels of a multi-level atomic system, which is, at certain times, observed when it interacts with a coherent electromagnetic field. This implies that a coherent electromagnetic field induces entanglement at the atomic level. Moreover, the generation of entanglement between two spatially 
separated clouds of particles is also possible [59]. Recent studies have demonstrated the occurrence of entanglement in particles at incredibly long distances from each other [60]. Moreover, entanglement may last for hours. It is reported that the nuclear spins of noble-gas atoms are exceptionally isolated from the environment and capable of maintaining their quantum properties for hours at room temperature [61].

\subsection{Entanglement of Neuron-like Objects in Different Systems}

Marletto et al. [62] attempted to discover empirical evidence of entanglement between Chlorobium tepidum bacteria (modeled as dipoles) and light (modeled as a single quantum harmonic oscillator). Recent studies demonstrated that the behavior of objects 15 micrometers in size was consistent with the laws of the quantum world, such as the phenomenon of quantum entanglement [63]. Moreover, an entangled state was reportedly generated between a millimeter-sized dielectric membrane and an ensemble of 109 atoms [64]. In comparison, a neuron's nucleus has a diameter of 3-18 micrometers, and a neuron is $4-100$ micrometers in size.

\subsection{Discussion}

The above-stated findings support the view that quantum mechanical mechanisms could contribute to goal-directed coherence in biological systems. This section discusses three possible agents that could involve quantum mechanisms within cells, thereby distributing the quality of goaldirected coherence in biological systems. Further research on quantum consciousness and the physical foundations of social interaction is limited unless such agents are explored. The concept of a protein as a candidate agent appears more plausible compared to the other two concepts presented above -the atom of hydrogen and the Posner molecule.

\section{Neurophysiological Foundation of Shared Intentionality}

In order to further assess the neurophysiological foundations of Shared Intentionality, the ordinary conditions of its appearance have to be determined. The Model of Coherent Intelligence proposes factors of interpersonal dynamics to be promoting coordinated neuronal processes in humans (Figure 1).

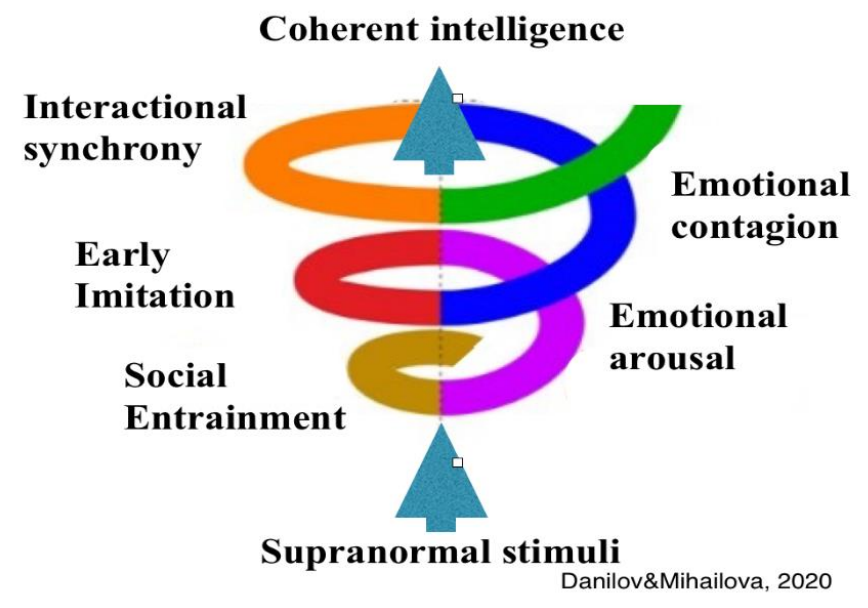

Figure 1 The sequence of appearances of Shared Intentionality during social dynamics. 
According to Danilov and Mihailova [5], continuing interpersonal dynamics (cyclically enhanced coordination of movements under ever-increasing arousal) create a coordinated mental process within groups.

A supranormal environment (for example, the first few hours of life) stimulates supranormal sensation in the mother-child dyads. It is possible that these continuing interpersonal dynamics push the inherited mechanism of social entrainment of the infants to the mother's rhythm (Figure 1). Both supranormal sensation and social entrainment may stimulate common emotional arousal. The continuing supranormal sensation and the infant's rhythm of arbitrary movements increase the emotional arousal in the dyad. The continuing supranormal sensation and the ever-increasing arousal in the dyad, as well as the rhythm of the infant's unintentional movements, together stimulate early imitation and emotional contagion [5]. These specific conditions of social dynamics specifically define the occurrence of the phenomenon of Shared Intentionality.

It appears that specific regions of the brain are engaged in shared sensory/cognitive processes irrespective of the 'valence of the feedback and the encoding of the subjective relevance of the feedback $[37,65]$. A common neural network is engaged in communicative intention processing independent of the modality used [66]. Other than the regions commonly involved in this processing, additional regions of the brain are engaged specifically according to the particular communicative modality [66]. According to Tettamanti et al. [67], the Intention Processing Network (IPN) involves the medial prefrontal cortex, precuneus, bilateral posterior superior temporal sulcus, and temporoparietal junctions.

According to the different social interaction modalities, the IPN is complemented by the activation of the additional regions of the brain, reflecting different Modality-Specific (M-S) input gateways to the IPN [67]. The M-S gateways mediate the structural and semantic decoding of the stimuli and provide the M-S information [67]. Sensory inputs of a specific modality are capable of activating the precise association of certain sensorimotor networks with specific emotion circuits in the brain [68].

The authors of the present study believe that the emotion-motion dynamics could cause the coordinated cognitive process of a high order in a group. Suppose that initially, the supranormal stimuli encourage implicit social dynamics among intimately-related individuals involved in social entrainment. In such a case, these organisms would experience common emotional arousal and could simultaneously bypass the interactional synchrony with the explicit manifestation of their emotion-motion coherence. Emotional arousal could elicit the evolutionary old circuits in the brain, which would interact with high-order cognitive and linguistic processing [68]. In parallel, the interactional synchrony stimulates a sensorimotor network, engaging the neural networks responsible for communicative intention processing (including high-order cognitive and linguistic processing), which are the precuneus, the left, and the right posterior STS and TPJ, and the medial pFC [66]. These two different experiences merge at high-order cognitive processing. The neural emotional networks and the sensorimotor networks are connected to various M-S gateways. The continuing upliftment of the interpersonal emotion-motion dynamics stimulates the intersects of the emotion-motion neural patterns in certain M-S gateways of each organism depending on (i) the pattern of the neural circuit engaged via emotional excitation and (ii) the pattern of the sensorimotor network [66]. The activation of these networks separately might not be adequately efficient for a tetanic stimulation of the neurons of all the M-S gateways connected to different emotional networks and sensorimotor networks. 
Therefore, the authors of the present study propose the hypothesis for describing how LongTerm Potentiation (LTP) could be induced specifically in particular M-S gateways (leaving the other gateways unstimulated even when various sensory factors stimulate all M-S gateways) while retaining information regarding the particular stimulus received (Figure 2). Although the ensemble of the emotion-motion integrated networks weakly stimulate the intersected neurons at their junction with the M-S gateways, if all the M-S gateways simultaneously receive weak stimulation from the receptors (due to the chaos of stimuli received by the pure nervous system), these multistimuli would contribute to the LTP in the particular M-S gateway that is at the junction of this emotion-motion ensemble due to the effect of synaptic cooperativity.

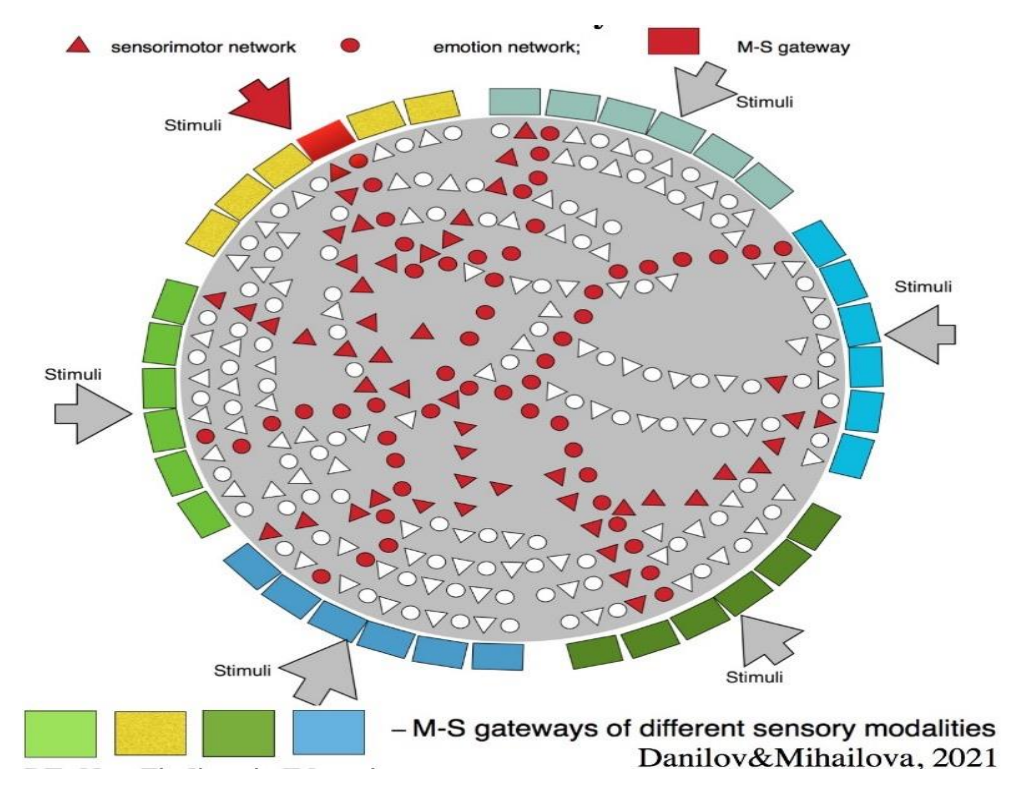

Figure $\mathbf{2}$ The schematic for the activation of specific M-S gateways.

Owing to the above-described continuing neurological coordinated activity, neurons from the M$S$ gateways at the junctions of the emotion and sensorimotor networks receive cooperative stimulation. Recent research has demonstrated that LTP could be induced cooperatively via the weaker stimulation of several pathways to a synapse [69], even though the ensemble of the emotion-motion integrated networks weakly stimulate the intersected neurons at their junction with the M-S gateways. Suppose all M-S gateways simultaneously receive weak stimulation from the receptors as well (due to the chaos of stimuli received by the pure nervous system). In such a case, this multi-signal would contribute to the LTP in the neurons of a particular M-S gateway at the junction of this emotion-motion ensemble due to the effect of the synaptic cooperativity because of the following reasons. LTP may be induced either by strong tetanic stimulation of a single pathway to a synapse or cooperatively via the weaker stimulation of several pathways. The neurons from the gateways at the junctions of these networks would receive cooperative stimulation. The induction of cooperativity would ensure the LTP.

According to Tazerart et al. [69], the synaptic cooperativity of only two neighboring synaptic inputs to the spines in the basal dendrites of $L 5$ pyramidal neurons extends the pre-post duration that could otherwise trigger potentiation. The engaged M-S gateways retain a specific stimulus, while the remaining M-S gateways (which are of the same sensory modality) remain unstimulated without retaining the information of the other stimuli. Therefore, specific M-S gateways are 
sensitive, and all of these organisms respond to specific sensory modalities. Figure 2 presents the schematic diagram of this process.

The induced emotion and sensorimotor networks (depicted in red in the schematic) activate particular M-S gateways even with weak stimulation of the sensory input. The M-S gateways depicted in different colors refer to different sensory modalities. At this point, the analysis encounters the foundation of the PDE problem -how do the immature neurons learn the timing code to modulate a particular synaptic strength, which then triggers either LTP or LTD in correspondence to the engagement of a set of specific stimuli, particularly the emotional and sensorimotor networks, as the structural organization of the excitatory inputs supporting spiketiming-dependent plasticity (STDP) remains unknown so far [69].

According to the received view, strong tetanic stimulation of a single pathway to a synapse generates LTP. Different regions of the brain exhibit different forms of LTP, and the types depend on several factors, such as age and the anatomic location of the neuron. However, the common processes are identical for all - the simple nature of Hebbian learning, which is based only on the coincidence of pre-and post-synaptic activity. Accordingly, LTP is persistent and lasts from several minutes to several months. It is this persistence that separates LTP from the other forms of synaptic plasticity (Abraham, 2003). STDP, which involves the pairing of the presynaptic and post-synaptic action potentials (APs), causes a variation of LTP or Long-Term Depression (LTD) [69]. The duration between the presynaptic and post-synaptic APs modulates the synaptic strength, thereby triggering either LTP or LTD [69]. A tetanic stimulation comprises a high-frequency sequence of individual stimulations of a neuron. The sign and magnitude of the change in the synaptic strength depend on the relative duration between the spikes of two connected neurons (the presynaptic and postsynaptic neurons) [69].

Next, one could ponder how are the neurons of an immature organism (even a newborn) able to learn the structural organization of the excitatory inputs that support STDP in relation to a complex comprising both sensory stimuli and the activation of certain sensorimotor and emotional networks of the nervous system.

The authors of the present study believe that LTP may be stimulated simultaneously in the neurons of certain M-S gateways by their entanglement state. A single harmonic oscillator during the continuing social dynamics of intimately-related organisms might induce the entanglement state of the neurons of certain M-S gateways in different nervous systems, thereby stimulating LTP in all of them simultaneously. The engaged M-S gateways of different organisms render these particular gateways relatively more sensitive to a certain stimulus, while the other M-S gateways of the same sensory modality remain depressed. This classification of stimuli into a single sensory modality is possible due to the significant number of M-S gateways within the brain that responds to a specific modality. Further arguments would demonstrate why the authors believe that the entanglement state of neurons could contribute to simultaneous LTP in neurons.

It appears uncontroversial to state that the pure nervous system in infants may experience emotions, although only primitive ones that are related to survival, such as hunger and pain. However, even though they possess inherited neuronal patterns of primitive emotional impressions, the newborns are unable to express themselves appropriately to a specific social case on their own. In addition, they cannot, alone and independently, understand the expression of other people's emotions [5, 26, 70]. The development of motor skills in them is also gradual. It is the daily routine that develops the neural patterns of primitive emotions and the sensorimotor neural 
patterns in infants. The everyday coherence of infants with the social world forms various integrated neural patterns of different emotions from the existing ensemble of scripts of emotions in their community. The authors believe that caregivers of infants contribute to the formation of these emotion scripts and consequently shape specific neural patterns in infants. It is evident that adults have experienced intentionality prior to the beginning of their coherent mental process with the newborns. Life experience has taught the adults particular emotion scripts, which have defined their precise motion kinematics and led to the formation of further elaborated sensorimotor patterns. In routine cooperation with a newborn, a caregiver enters into a coherent emotion-motion social dynamics with the newborn, who is under the influence of supranormal stimuli and in social entrainment. Therefore, similar M-S gateways are stimulated in the dyad.

While the immature nervous system of an infant meets with a chaos of stimuli, the adult's current intentionality has already stimulated particular networks that include the current emotion patterns and sensorimotor patterns. A part of them corresponds to a complex of primitive emotional and sensorimotor networks in the newborn with similar M-S gateways. These primitive networks in the newborn are less developed circuits, although these are similar to the part of the adult's wellintegrated complex network.

The induction of the timing-dependent LTP (t-LTP) and that of the t-LTD in single spines follow the bidirectional Hebbian STDP learning rule [69]. The Hebbian theory claims that an increase in synaptic efficacy arises from the learning process. If a single harmonic oscillator, during the continuing social dynamics of intimately-related organisms, induces the entanglement state of neurons of the certain M-S gateways in different nervous systems, and simultaneously, the adult neurons at the junctions of different emotion patterns and sensorimotor patterns receive the LTP, which are induced cooperatively via several stimulations, then the neurons of the mature organism train the neurons of the newborns as the neurons of both the adult organism and the newborn act together as a single unit because of being entangled. In the entanglement state, actions of the neurons of the mature organism determine and train the neurons of the newborn. The entanglement state of neurons is a possible mechanism through which the neurons of infants learn STDP. The entanglement state of these neurons ensures their immediate response to a specific stimulus regardless of the spatial division of organisms. Therefore, specific M-S gateways of both organisms are sensitive and respond equally to specific sensory modalities. The PDE problem in the chaos of stimuli requires a teaching mechanism. The entanglement state of neurons is also a possible mechanism underlying the learning of spike-timing-dependent plasticity in the neurons of infants. In cooperation of the adult with a blank mind, the emotion-motion coherence (Figure 1) ensures the induction of the same M-S gateways in neonates that are already involved in their respective caregivers (Figure 2 ). This involvement of similar networks and the sensibility of certain $\mathrm{M}-\mathrm{S}$ gateways because of their quantum entanglement last as long as necessary for teaching the immature nervous system.

\subsection{Discussion}

The present study proposed the neurophysiological and physical foundations of Shared Intentionality. The proposed hypothesis explains how Shared Intentionality in humans could emerge, bypassing perception. The core concept is that the neurons of a mature organism could teach the neonate neurons in the absence of communication between these organisms. According 
to the hypothesis of Shared Intentionality, such an interaction occurs in organisms during psychophysiological coherence. The mature neurons train the neonate's neurons in spike-timingdependent plasticity, linking particular stimuli with specific embodied sensorimotor neural networks. In particular, if a single harmonic oscillator induces the neurons (or Coherence Agent from these neurons) of modality-specific gateways in the organisms dwelling in a psychophysiological coherence, these neurons act coherently according to the laws of quantum mechanics. Since these neurons react similarly, the neurons of the mature organisms demonstrate to the neonate's neurons the appropriate response to high-frequency stimulation. These concepts demonstrate that Shared Intentionality could contribute to advanced digital modes of learning and Artificial Intelligence (AI) systems.

\section{Conclusion}

The present report discussed the physical foundations of the neurobiological process occurring during goal-directed coherence, i.e., which agent (element or compound) present in the cells is involved in coherent cooperation and how. The present study proposed concepts of physical foundations and the hypothesis of the neurophysiological process of social interaction in the absence of sensory cues referred to as Shared Intentionality. The core concept was that the neurons of a mature organism train the neonate's neurons in spike-timing-dependent plasticity, linking particular stimuli with specific embodied sensorimotor neural networks. In particular, if a single harmonic oscillator induces the neurons (or Coherence Agent from these neurons) of modalityspecific gateways in different organisms, which are in a psychophysiological coherence, these neurons act coherently according to the laws of quantum mechanics. Since these neurons react similarly, the neurons of the mature organism train the neurons of the neonate to exhibit the appropriate response to high-frequency stimulation by linking particular stimuli with specific embodied sensorimotor neural networks.

The present report discussed three possible agents that could involve quantum mechanisms within cells, thereby conferring the quality of goal-directed coherence to biological systems and promoting Shared Intentionality in humans. The concept of the protein agent appears to be more plausible compared to the other two - the atom of hydrogen and the Posner molecule. The report presented several comments to fabricate a framework for further research on the possible agents of quantum mechanics for goal-directed coherence and Shared Intentionality.

The report also presented several ideas for developing advanced online learning methods for children with developmental disadvantages. These ideas have been successfully tested in recent case studies (conducted online) [17, 18] concerning the task of conveying the meaning of numerosity to children of age 18-33 months. The core advantage of the present study is the concepts it proposes for further research on the neurophysiological process for understanding brain function at the cellular level during social interactions.

\section{Acknowledgments}

The author thanks reviewers who provided many valuable tips to make the paper more consistent and significant. 


\section{Author Contributions}

Igor Val Danilov formulated the hypothesis and wrote the first draft of the manuscript. Igor Val Danilov and Sandra Mihailova improved the text over several iterations.

\section{Funding}

No foundation that funded this research.

\section{Competing Interests}

The authors have declared that no competing interests exist.

\section{References}

1. Piaget J. The origins of intelligence in children. New York: Norton; 1963.

2. Chapman M. Intention, intentionality, and the constructive character of scientific knowledge. Psychol Inq. 1990; 1: 251-253.

3. Jones SS. The development of imitation in infancy. Philos Trans R Soc Lond B Biol Sci. 2009; 364: 2325-2335.

4. Jones S. Can newborn infants imitate? Wiley Interdiscip Rev Cogn Sci. 2017; 8: e1410.

5. Danilov IVal, Mihailova S. New findings in education: Primary data entry in shaping intentionality and cognition. Proceedings of The Thirteenth International Conference on Advanced Cognitive Technologies, and Applications COGNITIVE 2021; 2021 April 18th-22nd; Porto, Portugal. Wilmington: International Academy, Research, and Industry Association.

6. Thompson E. Mind in life: Biology, phenomenology, and the sciences of mind. 1st ed. Cambridge: The Belknap press of Harvard University press; 2010.

7. Delafield-Butt JT, Trevarthen C. Theories of the development of human communication [Internet]. Glasgow: The University of Strathclyde; 2012. Available from: https://strathprints.strath.ac.uk/39831/1/Delafield_Butt Trevarthen 2012 Theories of the development of human communication final edit 060312.pdf.

8. Csibra G, Gergely G. Natural pedagogy. Trends Cogn Sci. 2009; 13: 148-153.

9. Waxman SR, Leddon EM. Early word-learning and conceptual development [Internet]. Hoboken: Blackwell Publishing Ltd.; 2010 [cited 2021 March]. Available from: https://www.academia.edu/12821552/Early Word-Learning and Conceptual Development.

10. Shapiro L, Spaulding S. Embodied cognition. In: The Stanford encyclopedia of philosophy. Stanford: Stanford University; 2021.

11. Juarrero A. The self-organization of intentional action. Rev Int Philos. 2004; 228: 189-204.

12. Painter DR, Kim JJ, Renton Al, Mattingley JB. Joint control of visually guided actions involves concordant increases in behavioural and neural coupling. Commun Biol. 2021; 4: 816.

13. Fishburn FA, Murty VP, Hlutkowsky CO, MacGillivray CE, Bemis LM, Murphy ME, et al. Putting our heads together: Interpersonal neural synchronization as a biological mechanism for shared intentionality. Soc Cogn Affect Neurosci. 2018; 13: 841-849.

14. Danilov IVal, Mihailova S. Social interaction shapes infants' earliest links between language and cognition. Soc Sci Bull. 2019; 2: 145-157.

15. Danilov IVal, Mihailova S. Emotions in e-Learning: The review promotes advanced curriculum 
by studying social interaction. In: The 6th International Conference on Lifelong Education and Leadership Conference Proceeding Book. Turkey: Sakarya University, ICLEL; 2020. pp.8-17.

16. Danilov IVal, Mihailova S. Intentionality vs chaos: Brain connectivity through emotions and cooperation levels beyond sensory modalities. Proceedings of The Thirteenth International Conference on Advanced Cognitive Technologies, and Applications; 2021 April 18th-22nd; Porto, Portugal. Wilmington: International Academy, Research, and Industry Association.

17. Danilov IVal, Mihailova S, Reznikoff I. Frontiers in cognition for education: Coherent intelligence in e-learning for beginners aged 1 to 3 years. Proceedings of the 20th Int'I Conf on e-Learning, e-Business, Enterprise Information Systems, and e-Government, along with CSCE congress; 2021 July 26th-29th; Las Vegas, NV, USA. New York: American Council on Science and Health.

18. Danilov IVal, Mihailova S, Reznikoff I. "Shared intentionality in advanced problem based learning: Deep levels of thinking in coherent intelligence". Proceedings of the 17th Int'I Conf on Frontiers in Education: Computer Science and Computer Engineering, along with CSCE congress; 2021 July 26th-29th; Las Vegas, NV, USA. New York: American Council on Science and Health.

19. Commons ML, Trudeau EJ, Stein SA, Richards FA, Krause SR. Hierarchical complexity of tasks shows the existence of developmental stages. Dev Rev. 1998; 18: 237-278.

20. Danilov IVal, Mihailova S. Common origins of social interaction of different species: The model of coherent intelligence linking physics to social sciences". The proceedings of the CogSci 2021 conference, Comparative Cognition-Animal Minds; 2021 July 27th; Vienna, Austria. Wien: University of Vienna.

21. Danilov IVal. Contactless human-computer systems via shared intentionality: A concept design for the next generation of smart prosthetic limbs. In: Proceedings of the Future Technologies Conference. Cham: Springer; 2021. pp.776-791.

22. Zirbes L, Deneubourg JL, Brostaux Y, Haubruge E. A new case of consensual decision: Collective movement in earthworms. Ethology. 2010; 116: 546-553.

23. Ronacher B. Path integration in a three-dimensional world: The case of desert ants. J Comp Physiol A. 2020; 206: 379-387.

24. Buatois A, Lihoreau M. Evidence of trapline foraging in honeybees. J Exp Biol. 2016; 219: 24262429.

25. Danilov IVal. Ontogenesis of Social Interaction. J Med Clin Res Rev. 2020; 4: 1-7.

26. Danilov IVal. Social interaction in knowledge acquisition: Advanced curriculum. Critical review of studies relevant to social behavior of infants. Proceedings of The Twelfth International Conference on Advanced Cognitive Technologies and Applications COGNITIVE 2020; 2020 October 25th-29th; Nice, France. Wilmington: International Academy, Research, and Industry Association.

27. Tomasello $M$, Rakoczy $H$. What makes human cognition unique? From individual to shared to collective intentionality. Mind Lang. 2003; 18: 121-147.

28. Searle, JR, Slusser WS, Slusser M. Intentionality: An essay in the philosophy of mind. Cambridge: Cambridge University Press; 1983.

29. American Psychological Association. APA dictionary of psychology. Intention [Internet]. Washington: American Psychological Association. Available from: https://dictionary.apa.org/intention.

30. Lewis M. The development of intentionality and the role of consciousness. Psychol Inq. 1990; 1: 230-247. 
31. Bargh JA. Goal and intent: Goal-directed thought and behavior are often unintentional. Psychol Inq. 1990; 1: 248-251.

32. Searle JR. The Rediscovery of the Mind. London: MIT Press; 1992.

33. Tomasello M. Becoming human: A theory of ontogeny. Cambridge: Belknap Press of Harvard University Press; 2019.

34. Danilov IVal. Human-centered computing based on shared intentionality in human cognition model: A new approach to contactless human-computer system. Proceedings of the 23rd International Conference on Artificial Intelligence, along with CSCE congress; 2021 July 26th29th; Las Vegas, NV, USA. New York: American Council on Science and Health.

35. Schirmer A, Fairhurst $M$, Hoehl $S$. Being 'in sync'-ls interactional synchrony the key to understanding the social brain? Soc Cogn Affect Neurosci. 2021; 16: 1-4.

36. Valencia AL, Froese T. What binds us? Inter-brain neural synchronization and its implications for theories of human consciousness. Neurosci Conscious. 2020; 2020: niaa010.

37. Oldham S, Murawski C, Fornito A, Youssef G, Yücel M, Lorenzetti V. The anticipation and outcome phases of reward and loss processing: A neuroimaging meta-analysis of the monetary incentive delay task. Hum Brain Mapp. 2018; 39: 3398-3418.

38. Wass S, Haresign IM, Whitehorn M, Clackson K, Georgieva S, Noreika V, et al. Parental frontal brain activity tracks infants' attention during shared play. PsyArXiv. 2020. Doi: 10.31234/osf.io/u84dj.

39. Soon CS, Brass M, Heinze HJ, Haynes JD. Unconscious determinants of free decisions in the human brain. Nat Neurosci. 2008; 11: 543-545.

40. Fried I, Mukamel R, Kreiman G. Internally generated preactivation of single neurons in human medial frontal cortex predicts volition. Neuron. 2011; 69: 548-562.

41. Weingarten $C P$, Doraiswamy PM, Fisher $M$. A new spin on neural processing: Quantum cognition. Front Hum Neurosci. 2016; 10: 541.

42. André I, Bradley P, Wang C, Baker D. Prediction of the structure of symmetrical protein assemblies. Proc Natl Acad Sci USA. 2007; 104: 17656-17661.

43. Luo L, Lu J. Temperature dependence of protein folding deduced from quantum transition [Internet]. Ithaca: Cornell University; 2011. Available from:

https://arxiv.org/ftp/arxiv/papers/1102/1102.3748.pdf.

44. Baiesi M, Orlandini E, Seno F, Trovato A. Sequence and structural patterns detected in entangled proteins reveal the importance of co-translational folding. Sci Rep. 2019; 9: 8426.

45. Banerjee D. Energy transfer and entanglement in an optically active solution of amino acids. Ithaca: Cornell University; 2019. Available from:

https://arxiv.org/ftp/arxiv/papers/1909/1909.07795.pdf.

46. Sulkowska JI. On folding of entangled proteins: Knots, lassos, links and $\theta$-curves. Curr Opin Struct Biol. 2020; 60: 131-141.

47. Soltani Zangbar H, Ghadiri T, Vafaee MS, Ebrahimi Kalan A, Karimipour M, et al. A potential entanglement between the spinal cord and hippocampus: Theta rhythm correlates with neurogenesis deficiency following spinal cord injury in male rats. J Neurosci Res. 2020; 98: 24512467.

48. Storey C, Allan CY, Fisher PR. Phototaxis: Microbial. eLS. 2020. Doi: 10.1002/9780470015902.a0000399.pub3.

49. Losi A, Gaertner W. A light life together: Photosensing in the plant microbiota. Photochem 
Photobiol Sci. 2021; 20: 451-473.

50. Faini G, Del Bene F, Albadri S. Reelin functions beyond neuronal migration: From synaptogenesis to network activity modulation. Curr Opin Neurobiol. 2021; 66: 135-143.

51. Fisher MP. Quantum cognition: The possibility of processing with nuclear spins in the brain. Ann Phys. 2015; 362: 593-602.

52. Li XZ, Walker B, Michaelides A. Quantum nature of the hydrogen bond. Proc Natl Acad Sci USA. 2011; 108: 6369-6373.

53. Giudice ED, Tedeschi A. Water and autocatalysis in living matter. Electromagn Biol Med. 2009; 28: 46-52.

54. Giudice ED, Spinetti PR, Tedeschi A. Water dynamics at the root of metamorphosis in living organisms. Water. 2010; 2: 566-586.

55. Meijer DK, Jerman I, Melkikh AV, Sbitnev VI. Biophysics of consciousness: A scale-invariant acoustic information code of a superfluid quantum space guides the mental attribute of the universe. In: Rhythmic Oscillations in Proteins to Human Cognition. Singapore: Springer; 2021. pp.213-361.

56. Bandyopadhyay A, Ray K. Rhythmic oscillations in proteins to human cognition. Singapore: Springer; 2021.

57. Agarwal S, Aiello CD, Kattnig DR, Banerjee AS. The dynamical ensemble of the posner molecule is not symmetric. J Phys Chem Lett. 2021; 12: 10372-10379.

58. Streltsov A, Singh U, Dhar HS, Bera MN, Adesso G. Measuring quantum coherence with entanglement. Phys Rev Lett. 2015; 115: 020403.

59. Lange K, Peise J, Lücke B, Kruse I, Vitagliano G, Apellaniz I, et al. Entanglement between two spatially separated atomic modes. Science. 2018; 360: 416-418.

60. Yin J, Cao Y, Li YH, Liao SK, Zhang L, Ren JG, et al. Satellite-based entanglement distribution over 1200 kilometers. Science. 2017; 356: 1140-1144.

61. Katz O, Shaham R, Polzik ES, Firstenberg O. Long-lived entanglement generation of nuclear spins using coherent light. Phys Rev Lett. 2020; 124: 043602.

62. Marletto C, Coles DM, Farrow T, Vedral V. Entanglement between living bacteria and quantized light witnessed by Rabi splitting. J Phys Commun. 2018; 2: 101001.

63. Sillanpää M, Hong S. Spooky quantum entanglement goes big in new experiments [Internet]. Washington: Science News; 2018. Available from:

https://www.sciencenews.org/article/spooky-quantum-entanglement-goes-big-newexperiments.

64. Thomas RA, Parniak M, $\varnothing$ stfeldt C, Møller CB, Bærentsen C, Tsaturyan Y, et al. Entanglement between distant macroscopic mechanical and spin systems. Nat Phys. 2021; 17: 228-233.

65. Martins D, Rademacher L, Gabay AS, Taylor R, Richey JA, Smith DV, et al. Mapping social reward and punishment processing in the human brain: A voxel-based meta-analysis of neuroimaging findings using the Social Incentive Delay task. Neurosci Biobehav Rev. 2021; 122: 1-17.

66. Enrici I, Adenzato M, Cappa S, Bara BG, Tettamanti M. Intention processing in communication: A common brain network for language and gestures. J Cogn Neurosci. 2011; 23: 2415-2431.

67. Tettamanti M, Vaghi MM, Bara BG, Cappa SF, Enrici I, Adenzato M. Effective connectivity gateways to the theory of mind network in processing communicative intention. Neuroimage. 2017; 155: 169-176.

68. Nummenmaa L, Saarimäki H, Glerean E, Gotsopoulos A, Jääskeläinen IP, Hari R, et al. Emotional 
speech synchronizes brains across listeners and engages large-scale dynamic brain networks. Neurolmage. 2014; 102: 498-509.

69. Tazerart S, Mitchell DE, Miranda-Rottmann S, Araya R. A spike-timing-dependent plasticity rule for dendritic spines. Nat Commun. 2020; 11: 4276.

70. Danilov IVal. Emotions in learning towards coherent intelligence: The review of studies on social behavior in infants with visual impairment. J Med Clin Res Rev. 2020; 4: 1-6.

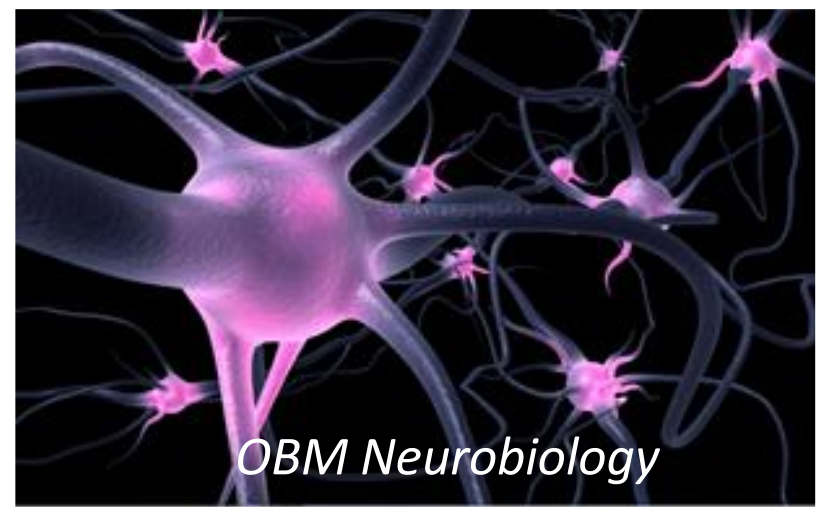

Enjoy OBM Neurobiology by:

1. Submitting a manuscript

2. Joining in volunteer reviewer bank

3. Joining Editorial Board

4. Guest editing a special issue

For more details, please visit:

http://www.lidsen.com/journals/neurobiology 